\title{
Management of Stroke: The Triumphs and the Travails
}

\author{
Robin Bhattarai ${ }^{1}$ \\ ${ }^{1}$ Department of Neurosurgery, B and C Medical College Teaching Hospital and Research Center, \\ Birtamode, Jhapa, Nepal. \\ Correspondence: \\ Dr. Robin Bhattarai, MS, PhD \\ Cerebrovascular \& Endovascular Neurosurgeon, \\ Department of Neurosurgery, \\ B and C Medical College Teaching Hospital and Research Center, Birtamode, Jhapa, Nepal. \\ Email: robinbhattarai@hotmail.com \\ Phone no: +9779842395698
}

$\longrightarrow$ erebrovascular diseases pose an immense requirement to understand the extremely complex structure of anatomical, physiological and pathological aspects of the human brain. This comes along with the need to understand the vascular-neural-endothelial-glial networks. These complexities impose as challenges to the understanding of "stroke".

Stroke is the third commonest cause of death in Nepal. According to the latest WHO data published in 2017 Stroke Deaths in Nepal reached 15,450 or $9.46 \%$ of total deaths. ${ }^{1}$ The age-adjusted Death Rate is 80.01 per 100,000 of the population. Earlier Jaya foundation ${ }^{2}$ in 2006 had an estimated 50,000 stroke every year however, Thapa et. ${ }^{3}$ in 2018 estimated about 1.16 million strokes occurred per year. This shows over a past decades stroke incidence has increased by 10 folds. Many of the stroke survivors have severe long-term disabilities.

Most of the hospitals in Nepal lack the necessary infrastructure and organization to quickly and effectively classify and treat stroke patients. We need to optimize clinical stroke services across the country to provide adequate stroke care. Existing treatment gaps in stroke care include a low thrombolysis rate; and the absence of $24 \times 7$ stroke physicians, endovascular neurosurgeons, stroke area maps, stroke care pathways, stroke units, stroke teams, adequate community awareness programs, and an effective public emergency system, all of which are essential elements for providing optimal stroke care to the community. It

61

Date submitted: 29/ 05/2020

Date accepted: 01/06/2020 is imperative to take immediate and appropriate measures to deal with the stroke in Nepal. $3,4,5,6,7$

Over the past decade, amazing progress has been made in understanding stroke management and outcomes. The treatment of stroke patients has changed from nihilism to hope. At the heart of this change is the tremendous advances in neuroimaging technology that have changed the way we assess stroke patients. In a few minutes, we can get information about the type of stroke, the cause, the vessels involved, the subtype, and accordingly give the best treatment which improves the prognosis and decreases the risk of recurrence.

The endovascular treatment of acute ischemic stroke (AIS) is a kind of therapy with obvious effect. Through timely and effective reperfusion, our hemiplegic and aphasic stroke patients can return to life and integrate into society. All recent trials, including MR CLEAN, EXTEND-1A, SWIFT-PRIME, REVASCAT, THRACE, THERAPY $8,9,10,11,12,13,14$ have given clear results, which are conducive to endovascular intervention in selective patients. Now, we have entered a new era of stroke treatment for severe AIS, aneurysm hemorrhage, and revascularization of large artery occlusion. Better equipment, better technology and better professional knowledge are all improving the therapeutic effect after stroke. Endovascular therapy has become a new nursing standard for AIS.

We will need to develop triage rules, systems and processes, and train new and existing emergency

egneuro, Volume 02, Issue 02, 2020 
personnel. We will need to assess the medical aspects of care, including the combination of thrombolytic drugs with intravascular thrombectomy, the use of anesthesia, adjuvant antithrombotic therapy, and medical management of blood pressure. We will need to identify the best and most appropriate image selection techniques to implement the best, time-sensitive management strategy for stroke treatment.

The "key" is "time with speed". and the only unified theme is "speed". From the Onset to reperfusion time is a new baseline process index, and it remains the basic principle of AIS.

As with intravenous recombinant tissue plasminogen activator, only a small proportion of stroke patients will need endovascular treatment, but this small part will promote the reorganization of the stroke care system.

It is important to design and enact the mandate of National Programs for Non-Communicable Diseases of Government of Nepal to address problems such as stroke burden in Nepal, what is unique to Nepal with regards to stroke in young patients, the risk factors, stroke in pregnancy, atherosclerotic patterns, stroke mortality and risk of recurrence, and, most importantly, management strategies that are best suited to Nepal's infrastructure and economy, to design protocols and blueprints to ensure optimal management of the majority of patients, regardless of socioeconomic and geographic barriers across the country.

Telestroke services in our country can integrate tertiary services with secondary and primary stroke care services, and extend to remote and poor areas of our society. We can start this kind of service by using low-cost smartphone technology and "Viber" social network. We can successfully establish an acute stroke care pathway and, under the guidance of trained emergency physicians, thrombolysis the patients with AIS at the district level without paying for it.

The ultimate goal of any treatment strategy is to maximize and ultimately restore normal function. Recently, the non-regenerative ability of the injured adult brain has been challenged, and neural plasticity has been recorded in experimental and clinical global and focal cerebral ischemia. Considering the fact that neuron circuits are complex arrays of neurons and connections, it is becoming more and more feasible and possible to enhance the possibility of reconstruction of damaged neuron networks through drugs, equipment, and robotics, which makes scientists in this field very happy to restorative medicine.

To quote Mark Twain, "we are at the best of times, and the worst of times". We are constantly challenged by the changing era; educating us with various ways to prevent, treat and rehabilitate stroke victims. Recovery from stroke can be rough but the triumph that follows is worth all the travails.

\section{References:}

1. HEALTH PROFILE NEPAL [Internet]. World Life Expectancy. Available from: https://www.worldlifeexpectancy.com/country-healthprofile/nepal

2. Pandit A, Arjyal A, Farrar J, Basnyat B. Nepal. Pract Neurol 2006; 6:129-33.

3. Thapa A, Kc B, Shakya B, Yadav DK, Lama K, Shrestha R. Changing Epidemiology of Stroke in Nepalese Population. Nepal J Neurosci. 2018 May 28;15(1):10-8.

4. Shaik MM, Loo KW, Gan SH. Burden of Stroke in Nepal. Int J Stroke. 2012 Aug;7(6):517-20.

5. Nepal G, Yadav JK, Basnet B, Shrestha TM, Kharel G, Ojha R. Status of prehospital delay and intravenous thrombolysis in the management of acute ischemic stroke in Nepal. BMC Neurol. 2019 Dec;19(1):155.

6. Br P. Stroke in Young Patients - A New Trend in Nepalese Perspective? 2015;3.

7. Bhalla D, Marin B, Preux PM. Stroke profile in Afghanistan and Nepal. Neurol Asia. 2009;8.

8. A Radiology Today staff report, Endovascular Stroke Treatment: 'MR CLEAN' Data Supports Within-the-Artery Approach to Treating Ischemic Stroke. Radiology Today 2015; 16:26.

9. Campbell B, Mitchell J, Kleinig TJ, Helen M, Desmond $\mathrm{PM}$, Geoffrey $\mathrm{AD}$, and Davis SM, for the EXTEND-IA Investigators. Endovascular Therapy for Ischemic Stroke with Perfusion-Imaging Selection. The New England Journal of Medicine 2015; 372:1009-18.

10. Jeffrey L, Goyal M, Bonafe A, Vivek K. Reddy, Rochemont RDM, Singer OC, Jahan R, for the SWIFT PRIME Stent-Retriever Thrombectomy after Intravenous tPA vs. t-PA Alone in Stroke. The New England Journal of Medicine 2015; 372:2285-95.

11. Smith WS. REVASCAT Trial: Further Advancement in Endovascular Stroke Therapy. Stroke 2015; 46:3012-13.

12. Ding Dale Endovascular Mechanical Thrombectomy for Acute Ischemic Stroke: A New Standard of Care. Journal of Stroke 2015; 17:123-6. 
Bhattarai $\mathbf{R}$ et al.

13. Bracard S, Ducrocq X. THRACE: Trial and cost effectiveness evaluation of intra-arterial thrombectomy in acute ischemic stroke; In: European stroke organisation (ESO) conference; 2015. Abstract 180.

14. Balasubramaian A, Mitchell P, Dowling R, Yan B. Evolution of Endovascular Therapy in Acute Stroke: Implications of Device Development. Stroke 2015; $17: 127-37$. 